\title{
Computational and experimental analyses of a liquid film flowing down a vertical surface
}

\author{
S. Sinkunas ${ }^{1}$, J. Gylys ${ }^{1} \&$ A. Kiela ${ }^{2}$ \\ ${ }^{1}$ Department of Thermal and Nuclear Energy, \\ Kaunas University of Technology, Lithuania \\ ${ }^{2}$ Department of Technical Sciences, Kaunas College, Lithuania
}

\begin{abstract}
The calculations evaluating velocity distribution across water and transformer oil film, and correspondingly the thickness of the film, are performed in this paper. Laminar plane film downward flow, and the film flowing on different convex surfaces of vertical tubes are explored. Equations for the calculation of local velocities in the film, with respect to cross curvature of wetted surface, were established. An evaluation of cross curvature influence on the film thickness is presented. An experimental study of velocity profiles for turbulent liquid film flow in the entrance region is performed as well. Analysis of profiles allowed estimating the length of stabilization for turbulent film flow under different initial velocities.
\end{abstract}

Keywords: velocity distribution, thickness of the film, laminar film, cross curvature, turbulent film, entrance region.

\section{Introduction}

The determination of hydromechanical parameters of liquid in falling film, emerging from a slit, is an interest in many applications of chemical, environmental and power engineering. The precise prediction of internal or external film flow plays an important role in the design of heat exchangers.

The minimum total energy criteria for determining the minimum wetting rate, and the minimum thickness of an isothermal thin liquid film flowing down a vertical adiabatic surface, was examined in [1]. An analytical expression of the profile of a stable liquid rivulet and of two-dimensional velocity distribution in the rivulet was developed. 
Instantaneous velocity profiles across a wavy laminar film and axial profiles of thickness were measured simultaneously and statistically analysed in [2]. Experimental data indicated that the time-averaged mean and maximum velocity data are significantly over-predicted by Nusselt's theory, while the timeaveraged film thickness data are slightly under-predicted.

The velocity distribution of the falling film was investigated [3]. The cylindrical model appeared to be more appropriate over the Cartesian model when the film thickness to tube diameter ratio is large. The study showed that the wave characteristics depend on the parameters such as dimensionless wave velocity, tube radius and Reynolds number.

Experimental data on falling film characteristics have been obtained in [4]. These data suggest that the tube diameter strongly affects film flow development, possibly promoting wave interaction and damping. At small liquid Reynolds numbers, critical flooding velocities decrease with increasing liquid rate.

Dimensionless empirical models for the average thickness and radial velocity of wavy films based on thickness measurements on a rotating cone surface were developed [5]. The proposed models express the film thickness and radial velocity as functions of cone geometrical and operating parameters.

Experiments [6] were performed to investigate the flow and surface structure in laminar wavy films over Reynolds numbers from 27 to 200. Measurements of the velocity and film thickness by a fluorescence technique enabled detailed information on the transient conditions within the three-dimensional wavy flow to be gained.

Film velocity and thickness measurements were made in wavy laminar films falling on the exterior of a vertical column [7]. Examination of these conditions revealed that large waves behave as lumps of liquid sliding over a continuous substrate. Velocity fields within substrates displayed reduced sensitivity to the large waves with increases in substrates.

Analytical and experimental study of velocity profiles for laminar and turbulent liquid film flow on vertical surface was the purpose of the present work.

\section{Analytical method}

\subsection{Influence of a wetted surface cross curvature on the thickness of laminar film}

Let's consider a stabilized laminar film flow on the external surface of a vertical tube and on a vertical plane surface respectively. A stabilized gravity film flow is assumed when the film thickness and average velocity do not change in flow direction. In this case the thickness of the boundary layer is equal to the film thickness.

The major contribution to the analysis of falling down laminar film is Nusselt's solution of the motion equation 


$$
\frac{d^{2} w}{d y^{2}}+\frac{g}{v}\left(1-\frac{\rho_{g}}{\rho}\right)=0
$$

with boundary conditions

$$
w=0, \text { for } y=0 ; \frac{d w}{d y}=0, \text { for } y=\delta .
$$

In the case of stabilized gravity film flow often is $\rho_{g}<<\rho$, so the member $1-\rho_{g} / \rho$ can be ignored. Solving eqn (1), one can obtain parabolic equation of velocity for laminar film

$$
w=\frac{g y \delta}{v}\left(1-0.5 \frac{y}{\delta}\right) .
$$

By integrating eqn (3) within the limits from 0 to $\delta$, we obtain the expression of mean velocity for laminar film flow on vertical plane surface

$$
\bar{w}=\frac{g \delta^{2}}{3 v}=\left(\frac{g v}{48} R e^{2}\right)^{1 / 3}
$$

Multiplication of eqn (4) by $\delta \rho$ leads to the formula defining the thickness of laminar film

$$
\delta_{p l}=\left(\frac{3 \Gamma v}{\rho g}\right)^{1 / 3}=\left(\frac{3}{4} \frac{v^{2}}{g} \operatorname{Re}\right)^{1 / 3}
$$

Equation of motion for the liquid film in cylindrical coordinates is as follows

$$
\frac{d^{2} w}{d r^{2}}+\frac{1}{r} \frac{d w}{d r}+\frac{g}{v}=0
$$

By working out eqn (6) with the following boundary conditions

$$
w=0, \text { for } r=R ; \frac{d w}{d r}=0, \text { for } r=R+\delta,
$$

we obtain velocity distribution across the film 
342 Computational Methods in Multiphase Flow IV

$$
w=\frac{g(R+\delta)^{2}}{2 v} \ln \frac{r}{R}-\frac{g}{4 v}\left(r^{2}-R^{2}\right)
$$

or

$$
w=\frac{g R^{2}}{2 v}\left[\left(1+\varepsilon_{R}\right)^{2} \ln \frac{r}{R}-0.5\left(\frac{r^{2}}{R^{2}}-1\right)\right] .
$$

The mean velocity for film flow on the external tube surface we can express as

$$
\bar{w}=\frac{\int_{R}^{R+\delta} w d r}{\int_{R}^{R+\delta} r d r}
$$

and identify the following relation

$$
\bar{w}=\frac{g R^{3}}{4 v\left(1+0.5 \varepsilon_{R}\right) \delta}\left\{\left(1+\varepsilon_{R}\right)^{4}\left[\ln \left(1+\varepsilon_{R}\right)-0.75\right]+\left(1+\varepsilon_{R}\right)^{2}-0.25\right\} .
$$

Mass flow rate of liquid film is defined as

$$
G=\bar{w} \rho f=2 \pi R \bar{w} \rho \delta\left(1+0.5 \varepsilon_{R}\right)
$$

and by substituting eqn (11) for eqn (12), we get

$$
G=\frac{\pi \rho g R^{4}}{2 v}\left\{\left(1+\varepsilon_{R}\right)^{4}\left[\ln \left(1+\varepsilon_{R}\right)-0.75\right]+\left(1+\varepsilon_{R}\right)^{2}-0.25\right\} .
$$

Defining Reynolds number for film flow as

$$
R e=\frac{4 G}{2 \pi R \rho v}
$$

and substitution of eqn (13) by eqn (14) leads to the following expression

$$
R e=\frac{g \delta^{3}}{\varepsilon_{R}^{3} v}\left\{\left(1+\varepsilon_{R}\right)^{4}\left[\ln \left(1+\varepsilon_{R}\right)-0.75\right]+\left(1+\varepsilon_{R}\right)^{2}-0.25\right\}
$$


From eqn (15) thickness of the film may be evaluated as follows

$$
\delta=\varepsilon_{R}\left(\frac{v^{2}}{g} R e\right)^{1 / 3}\left\{\left(1+\varepsilon_{R}\right)^{4}\left[\ln \left(1+\varepsilon_{R}\right)-0.75\right]+\left(1+\varepsilon_{R}\right)^{2}-0.25\right\}^{-1 / 3} .
$$

By solving a set of eqns (5) and (16), we obtain the expression defining the thickness of the film flowing down a convex surface

$$
\delta=\delta_{p l} \frac{\varepsilon_{R}}{\left\{0.75\left[\left(1+\varepsilon_{R}\right)^{4}\left(\ln \left(1+\varepsilon_{R}\right)-0.75\right)+\left(1+\varepsilon_{R}\right)^{2}-0.25\right]\right\}^{1 / 3}},
$$

where curvature coefficient which corrects film thickness for cross curvature of the film can be defined as

$$
C_{R \delta}=\frac{\varepsilon_{R}}{\left\{0.75\left[\left(1+\varepsilon_{R}\right)^{4}\left(\ln \left(1+\varepsilon_{R}\right)-0.75\right)+\left(1+\varepsilon_{R}\right)^{2}-0.25\right]\right\}^{1 / 3}} .
$$

Finally, the thickness of laminar gravitational liquid film on vertical convex surface can be calculated as

$$
\delta=\left(\frac{3}{4} \frac{v}{g} \operatorname{Re}\right)^{1 / 3} C_{R \delta}
$$

Eqn (18) for the calculation of curvature correction coefficient is sufficiently complicated, so the following equation of a simple form has been developed

$$
C_{R \delta}=\left(1+0.3 \varepsilon_{R}\right)^{-1}
$$

Practically the case with values or relative cross curvature exceeding 1 is not significant. Therefore, eqn (19) with sufficient accuracy can be simplified

$$
\delta=\frac{\delta_{p l}}{1+0.3 \varepsilon_{R}}
$$

In engineering calculations the cross curvature of wetted surface is usually known and the thickness of the film flowing down a vertical plane surface one can estimate from eqn (5). Then, the equation describing the film flowing down the convex surface results from eqn (21)

$$
\delta=1.67 R\left(\sqrt{1+1.2 \delta_{p l} / R}-1\right) .
$$


By substituting eqn (5) for eqn (22) we obtain

$$
\delta=1.67 R\left(\sqrt{1+1.09\left(R e / G a_{R}\right)^{1 / 3}}-1\right) .
$$

As it was mentioned above, in practice the relative cross curvature of the film is $\varepsilon_{R}<1$, so it is more convenient to estimate the relative cross curvature from the following equation

$$
\varepsilon_{R}=1.67\left(\sqrt{1+1.09\left(R e / G a_{R}\right)^{1 / 3}}-1\right)
$$

and then the film thickness

$$
\delta=\varepsilon_{R} R
$$

The above-discussed method was applied for the calculations of velocity distribution in transformer oil film. The kinematic viscosity of transformer oil was equal $v=27.8 \times 10^{-6} \mathrm{~m}^{2} / \mathrm{s}$ at $20^{\circ} \mathrm{C}$ temperature. Three values of Reynolds numbers were chosen for the computation. The calculations were performed for the liquid film flowing down a plane and convex vertical surfaces correspondingly with different outside diameters of vertical tubes ( 3 and $30 \mathrm{~mm}$ diameters).

The results of calculations are shown in figure 1. As we can see from figure 1 , the influence of cross curvature for the liquid film of high viscosity is noticeable.

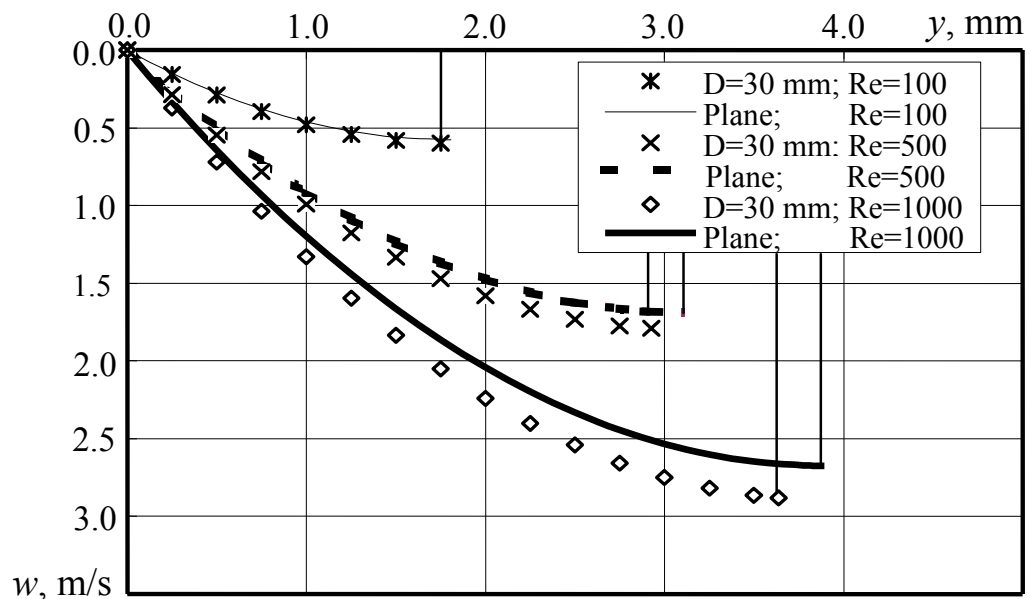

Figure 1: Local velocity profiles of transformer oil film flowing down a pane and convex surfaces. 


\section{Experimental method}

\subsection{Experimental set-up}

In order to measure velocity profiles of turbulent film a special device (figure 2) was applied. Velocity profiles in water film were measured by a needle probe, which was $0.3 \mathrm{~mm}$ in outside diameter and $0.18 \mathrm{~mm}$ in inside diameter. A coordinating mechanism carried out the movement of the needle probe and fixed its zero position with respect to the wall. The needle probe was connected to a vertical glass tube through the flexible tube. Dynamic pressure in the needle probe was determined by the height of the water column in the glass tube. In order to increase the accuracy of readings, a clock-faced indicator was fixed to the coordinating mechanism.

The location of the velocity measuring point in the water film was determined by the following formula

$$
y=y_{n}+0.1 d \text {. }
$$

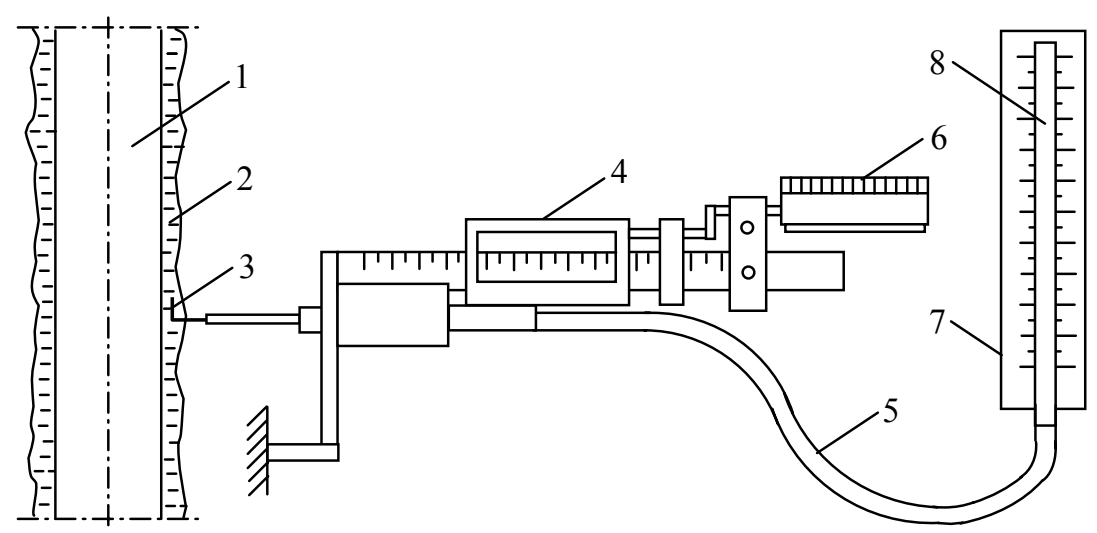

Figure 2: Device to measure velocity profiles of water film: 1 - wetted tube; 2 - water film; 3 - needle probe; 4 - coordinating mechanism; 5 - flexible tube; 6 - clock-faced indicator; 7 - scale; 8 - glass tube.

\subsection{Velocity profiles in the entrance region of a turbulent film}

The entrance region of a film flow is fixed when the film average thickness and average velocity becomes stable. The entrance region can be determined by the function $\varphi=f(\eta)$ provided that dynamic velocity $v^{*}$ is calculated the regularities of stabilized flow and local velocity of the film is real. In that way, 
defined velocity profiles take up a position above or below profiles of stabilized flow depending on film velocity in the entrance region of film flow. It evidently is seen from figures 3 and 4, where measured profiles in the entrance region of turbulent film flow are presented. As we can see from figures 3 and 4, the initial length of film flow depends on parameter $\varepsilon=\bar{w}_{d} / \bar{w}_{\text {stab }}$ and wetting density $(R e)$. In this case the film's mean velocity in the liquid distributor was calculated as follows

$$
\bar{w}_{d}=G / f_{d} \rho \text {. }
$$

The film mean velocity for stabilized flow has been calculated from the following equation

$$
\bar{w}_{\text {stab }}=1.85(g v)^{1 / 3} \operatorname{Re}^{5 / 12} .
$$

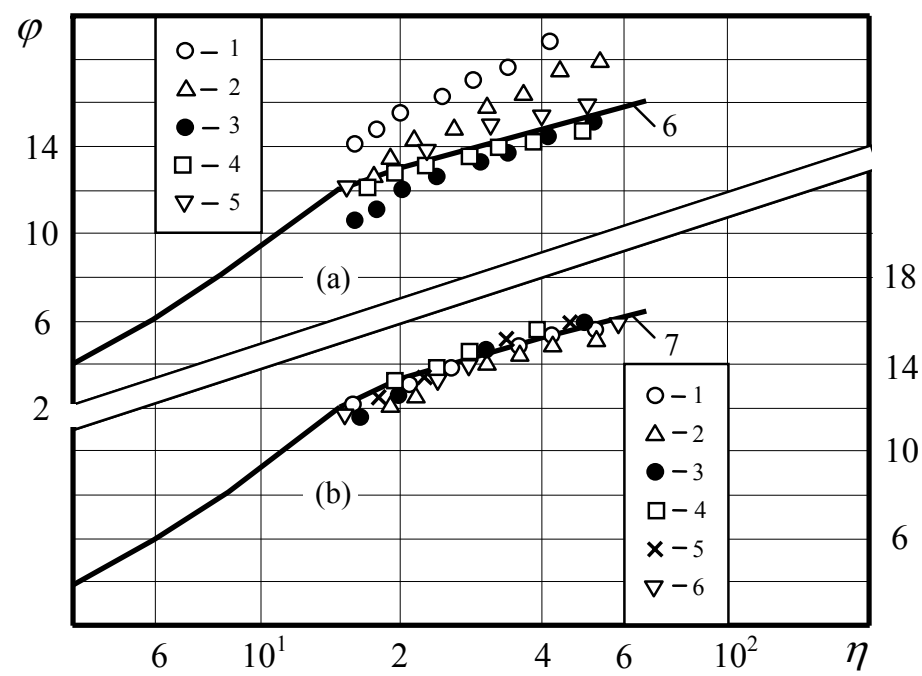

Figure 3: Velocity profiles of water film in the entrance region of film flow when $\operatorname{Re}_{c r}<\operatorname{Re}<\operatorname{Re}_{t}$ : a) $1-\operatorname{Re}=5.7 \cdot 10^{3}, \varepsilon=1.46, x=0.08 ; 2-\operatorname{Re}$ $=8.16 \cdot 10^{3}, \varepsilon=1.2, x=0.08 ; 3-R e=5.84 \cdot 10^{3}, \varepsilon=0.89, x=0.08$; $4-\operatorname{Re}=4.87 \cdot 10^{3}, \varepsilon=1.48, x=0.28 ; 5-\operatorname{Re}=5.25 \cdot 10^{3}, \varepsilon=0.91, x$ $=0.28 ; 6$ - theoretical calculation, stabilized flow, $\operatorname{Re}=5.05 \cdot 10^{3}$; b) $1-\operatorname{Re}=6.2 \cdot 10^{3}, \varepsilon=1.44, x=0.48 ; 2-\operatorname{Re}=7.76 \cdot 10^{3}, \varepsilon=1.21, x$ $=0.48 ; 3-\operatorname{Re}=6.43 \cdot 10^{3}, \varepsilon=0.87, x=0.48 ; 4-\operatorname{Re}=5.43 \cdot 10^{3}$, $\varepsilon=1.46, x=1.07 ; 5-\operatorname{Re}=6.19 \cdot 10^{3}, \varepsilon=1.28, x=1.07 ; 6-\operatorname{Re}=$ $5.5 \cdot 10^{3}, \varepsilon=0.90, x=1.07 ; 7-$ theoretical calculation, stabilized flow, $\operatorname{Re}=5.05 \cdot 10^{3}$. 


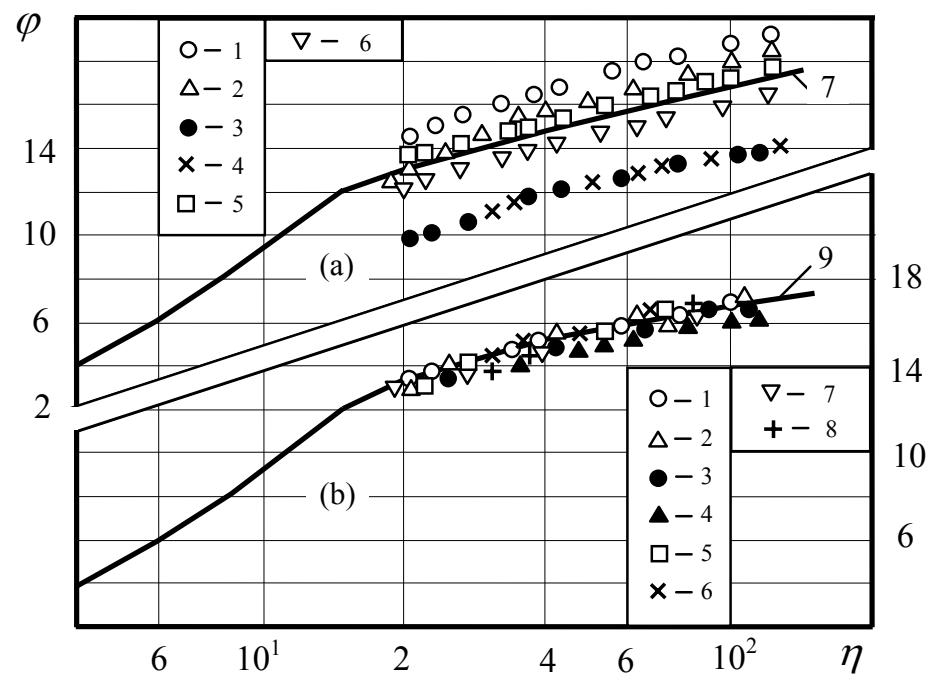

Figure 4: Velocity profiles of water film in the entrance region of film flow when $\operatorname{Re}>\operatorname{Re}_{t}$ : a) $1-\operatorname{Re}=15.8 \cdot 10^{3}, \varepsilon=1.14, x=0.08 ; 2-\operatorname{Re}=$ $13.2 \cdot 10^{3}, \varepsilon=0.94, x=0.08 ; 3-\operatorname{Re}=16 \cdot 10^{3}, \varepsilon=0.69, x=0.08$; $4-\operatorname{Re}=41.8 \cdot 10^{3}, \varepsilon=0.78, x=0.08 ; 5-\operatorname{Re}=15.8 \cdot 10^{3}, \varepsilon=1.14, x$ $=0.28 ; 6-\operatorname{Re}=13.9 \cdot 10^{3}, \varepsilon=0.72, x=0.28 ; 7-$ theoretical calculation, stabilized flow, $R e=35 \cdot 10^{3}$; b) $1-\operatorname{Re}=15.9 \cdot 10^{3}, \varepsilon=$ $1.14, x=0.48 ; 2-\operatorname{Re}=16.3 \cdot 10^{3}, \varepsilon=1.0, x=0.48 ; 3-\operatorname{Re}=15 \cdot 10^{3}$, $\varepsilon=0.69, x=0.48 ; 4-\operatorname{Re}=37 \cdot 10^{3}, \varepsilon=0.82, x=0.48 ; 5-\operatorname{Re}=$ $14.6 \cdot 10^{3}, \varepsilon=1.16, x=1.07 ; 6-R e=13.7 \cdot 10^{3}, \varepsilon=1.04, x=1.07$; $7-\operatorname{Re}=13.8 \cdot 10^{3}, \varepsilon=0.71, x=1.07 ; 8-\operatorname{Re}=36.8 \cdot 10^{3}, \varepsilon=0.82, x$ $=0.71 ; 9-$ theoretical calculation, stabilized flow, $R e=35 \cdot 10^{3}$.

\section{Conclusions}

It is evident that under the influence of convex surface curvature the film thickness decreases changing local velocity distribution simultaneously. An equation for the calculation of local velocities in the film with a wetted surface curvature has been established.

Physical properties of the liquid, especially its viscosity, have greater influence on hydromechanical parameters of the film than cross curvature of the wetted surface.

The initial velocity and wetting density has a significant influence on the length of stabilization for turbulent film flow. It is obtained that film flow stabilization takes place at the distance $0.5 \mathrm{~m}$ when $6 \cdot \mathrm{E} 3<=R e<=7 \cdot \mathrm{E} 3$ and at $1.0 \mathrm{~m}$ when $\operatorname{Re}<4 \cdot \mathrm{E} 4$. 


\section{Nomenclature}

$D$ - tube diameter, $\mathrm{m} ; d$ - needle probe outside diameter, $\mathrm{m} ; f$ - cross sectional area of film flow, $\mathrm{m}^{2} ; G$ - liquid mass flow rate, $\mathrm{kg} / \mathrm{s}$; $g$ - acceleration of gravity, $\mathrm{m} / \mathrm{s}^{2} ; G a_{R}$ - Galileo number, $g R^{3} / v^{2} ; R$ - tube external radius, $\mathrm{m} ; r$ - variable radius, $\mathrm{m} ; R e$ - Reynolds number of liquid film, $4 \Gamma /(\rho v) ; v^{*}$ - dynamic velocity, $\left(\tau_{w} / \rho\right)^{1 / 2}, \mathrm{~m} / \mathrm{s} ; w-$ local velocities of stabilized film, $\mathrm{m} / \mathrm{s} ; \bar{w}$ - average velocities of stabilized film, $\mathrm{m} / \mathrm{s} ; y$-distance from wetted surface, $\mathrm{m} ; y_{n}$ - distance from needle probe centre to the wall; $\Gamma$ - wetting density, $\mathrm{kg} /(\mathrm{ms}) ; \delta$ - liquid film thickness, $\mathrm{m} ; \varepsilon$ - relative film velocity, $\bar{w}_{d} / \bar{w}_{\text {stab }} ; \varepsilon_{R}-$ relative cross curvature of the film, $\delta / R$; $\eta$ - dimensionless distance from the wetted surface, $y / \delta ; v$ - kinematic viscosity, $\mathrm{m}^{2} / \mathrm{s} ; \rho$ - liquid density, $\mathrm{kg} / \mathrm{m}^{3} ; \varphi$ - dimensionless film velocity, $w / v^{*} ; \tau_{w}-$ shear stress at the wall, Pa;

Subscripts: $c r$ - critical; $d$ - distributor; $g$ - gas or vapour; $p l$ - plane surface; stab - stabilized flow; $t$-turbulent; $w$ - wetted surface.

\section{References}

[1] El-Genk, M.S. \& Saber, H.H., Minimum thickness of a flowing down liquid film on a vertical surface. International Journal of Heat and Mass Transfer, 44(15), pp. 2809-2825, 2001.

[2] Moran, K., Inumaru, J. \& Kawaji, M., Instantaneous hydrodynamics of a laminar wavy liquid film. International Journal of Multiphase Flow, 28(5), pp. 731-755, 2002.

[3] Kil, S.H., Kim, S.S. \& Lee, S.K., Wave characteristics of falling film on a vertical circular tube. International Journal of Refrigeration, 24(6), pp. 500-509, 2001.

[4] Mouza, A.A., Paras, S.V. \& Karabelas, A.J., The influence of small tube diameter on falling film and flooding phenomena. International Journal of Multiphase Flow, 28(8), pp. 1311-1331, 2002.

[5] Makarytchev, S.V., Langrish, T.A.G. \& Prince, R.G.H., Thickness and velocity of wavy liquid films on rotating conical surfaces. Chemical Engineering Science, 56(1), pp. 77-87, 2001.

[6] Adomeit, P. \& Renz, U., Hydrodynamics of three-dimensional waves in laminar falling films. International Journal of Multiphase Flow, 26(7), pp. 1183-1208, 2000.

[7] Mudawar, I. \& Houpt, R.A., Measurement of mass and momentum transport in wavy-laminar falling liquid films. International Journal of Heat and Mass Transfer, 36(17), pp. 4151-4162, 1993. 